\title{
Leishmaniasis Visceral Canina: Pruebas Diagnósticas no identifican Estados Reales de la Infección
}

\section{Canine Visceral Leishmaniasis: Diagnostic tests do not detect real state of the infection}

\author{
Marlyn H. Romeroํㅜ, Myriam C. López ${ }^{2}$, María C. Echeverry² y Favio A. Rivas² \\ 1 Departamento de Salud Animal, Facultad de Ciencias Agropecuarias, Universidad de Caldas. \\ Manizales, Colombia. marlyn.romero@ucaldas.edu.co \\ 2 Departamento de Salud Pública, Facultad de Medicina, Universidad Nacional de Colombia, \\ Bogotá.mclopezp@unal.edu.co,mcecheverryg@unal.edu.co, farivasm@unal.edu.co
}

Recibido 26 Julio 2007/Enviado para Modificación 28 Enero 2008/Aceptado 10 Abril 2008

\section{RESUMEN}

Objetivos Establecer la capacidad de las pruebas serológicas de Inmunofluorescencia indirecta (IFAT) e Inmunoensayo ligado a enzimas (ELISA), para detectar el estado real de la infección en leishmaniasis visceral canina (LVC). Métodos Un total de 211 perros criollos localizados en el sur del Departamento del Tolima, zona endémica para la leishmaniasis visceral, fueron evaluados por medio de examen clínico y serológico, usando como antígeno la cepa colombiana de Leishmania infantum (infantum) MHOM/COL/CL044B. Así mismo, con la finalidad de establecer reacciones cruzadas o coinfecciones, se evaluó la reactividad de los sueros ante antígenos específicos de Trypanosoma cruzi, por medio de la prueba Western Blot (WB). La frecuencia de LVC fue del 44,1 \% (93/211) y 50,2 \% (103/211) mediante IFAT y ELISA respectivamente.

Resultados La seroreactividad ante antígenos específicos de $T$. cruzi fue del 1,42 $\%(3 / 211)$. La concordancia de las técnicas serológicas fue baja (K=12,1\%) y a pesar de la presencia de signos clínicos en los animales evaluados, las razones de prevalencia halladas, demostraron que no hay asociación entre la ocurrencia de las manifestaciones clínicas y la seropositividad a las pruebas diagnósticas.

Conclusiones La baja asociación de las pruebas serológicas utilizadas en el diagnóstico de Leishmania infantum, sugiere que es necesario desarrollar estudios que permitan establecer un algoritmo de pruebas diagnósticas en el país para confirmar el estado real de la infección de los animales y de esta forma orientar eficientemente los recursos de Salud Pública destinados al control de la enfermedad.

Palabras Clave: Leishmania, prueba ELISA, diagnóstico serológico (fuente: DeCS, BIREME). 


\begin{abstract}
Objectives Establishing indirect immunofluorescence (IFAT) and enzyme-inked immunoassay (ELISA) serological tests' ability to detect canine visceral leishmaniasis (CVL) infection.

Methods 211 cross-bred dogs from the south of the Tolima department (a visceral leishmaniasis endemic area) were evaluated by clinical and serological exams, using the Colombian Leishmania infantum (infantum) MHOM/COL/CL044B strain as antigen. Sera reactivity to Trypanosoma cruzi-specífic antigens was evaluated by Western blot (WB) for establishing crossed-reactions or coinfections. CVL frequency was $44,1 \%(93 / 211)$ by IFAT and 50,2 \% (103/211) by ELISA.

Results Seroreactivity to T. cruzi-specific antigens was 1,42 \% (3/211). Agreement between the serological techniques was low $(K=12,1 \%)$ and, in spite of clinical signs being present in the animals being evaluated, the prevalence ratios found demonstrated the lack of association between the occurrence of the clinical manifestations and the diagnostic tests' seropositivity.

Conclusions The low association between the serological tests used in diagnosing Leishmania infantum suggested the need for further studies aimed at establishing an algorithm for diagnostic tests in Colombia for confirming the real state of the animals' infection and thereby efficiently orientating public health resources allocated for controlling canine visceral leishmaniasis.
\end{abstract}

Key Words: Canine visceral leishmaniasis, IFAT, ELISA, serological tests (source: $\mathrm{MeSH}, \mathrm{NLM}$ ).

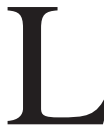

a Leishmaniasis Visceral (LV) es una enfermedad zoonótica causada por un protozoo de la familia Trypanosomatidae, conocido como Leishmania infantum en el nuevo mundo. Es transmitida por la picadura de vectores flebotomíneos del género Lutzomyia sp. (1-3). Los cánidos (Canis familiares) son los principales reservorios domésticos de la LV y son considerados responsables de la presentación endémica/epidémica natural de la enfermedad, sugiriéndose que el hombre sería una fuente de infección secundaria para los vectores y la transmisión dependería básicamente de la presencia de caninos infectados $(1,5,6)$.

Tradicionalmente los métodos de control de la LV se han basado en el tratamiento de las personas afectadas, en la disminución de la densidad del vector e identificación y eliminación de caninos infectados $(1,7)$. En los programas de Salud Pública en el mundo, se han utilizado las pruebas serológicas para la identificación de animales infectados; teniendo en cuenta que el diagnóstico clínico de la leishmaniasis visceral canina (LVC) es difícil porque la sintomatología es variable e inespecífica $(1,8,9)$; así mismo, el diagnóstico defi- 
nitivo depende del aislamiento parasitológico en cultivos o por detección del DNA del parásito por PCR (8). Las técnicas anteriores son costosas, invasivas, requieren de tiempo, personal calificado y equipos costosos $(1,8)$.

En Colombia, la detección de anticuerpos contra L. infantum en caninos se ha llevado a cabo tradicionalmente mediante Inmunofluorescencia Indirecta (IFAT) $(10,11)$. En el año 2003 se estandarizó una prueba de ELISA, que ha sido descrita como más sensible y menos subjetiva que IFAT (10). El presente estudio se realizó con el propósito de examinar si las pruebas diagnósticas usadas en Colombia (IFAT y ELISA) permiten establecer el estado real de la infección en animales localizados en zonas endémicas colombianas.

\section{MÉTODOS}

Área geográfica

El área de estudio se localiza en el sur del departamento del Tolima y está conformada por los municipios de Coyaima, Natagaima, Purificación, Ataco, Coello y Ortega, donde anualmente se reportan casos humanos de LV. Se caracterizan por ser zonas climáticas variadas, donde hay bosque seco tropical, bosque húmedo premontano y bosque muy húmedo montano bajo. Los municipios se encuentran a una altitud que fluctúa entre 323 y 1445 m.s.n.m. (12).

\section{Muestra}

Se analizaron doscientos once sueros caninos que fueron recolectados en la zona endémica del LV en el departamento del Tolima, durante el año 2005. Se obtuvo el consentimiento informado de los propietarios de los animales para realizar el examen clínico y la toma de muestras de sangre, siguiendo las normas éticas para el manejo de animales establecidas en la Ley 84 de 1989 y las normas éticas para investigación sin riesgos en comunidades, descritas en la resolución del Ministerio de Salud No. 8430 de 1993. Se recolectó sangre completa por venopunción para la obtención de suero y posterior análisis.

\section{Examen clínico}

De acuerdo a su condición clínica los animales fueron clasificados como asintomáticos, cuando no presentaron ningún signo clínico compatible con la infección; oligosintomáticos (uno o dos síntomas) y polisintomáticos (más de dos síntomas), como ha sido descrito por Travi (10). 


\section{IFAT}

La técnica fue desarrollada siguiendo la metodología descrita por Corredor (13). Como antígeno se emplearon promastigotes en fase metacíclica de Leishmania infantum (infantum) cepa MHOM/COL/CL-044B. Se consideraron como títulos positivos aquellos iguales o superiores a 1:32.

\section{ELISA}

En la detección de anticuerpos por ELISA, se utilizó el antígeno cepa MHOM/ COL/CL-044B , siguiendo la metodología descrita por Vega (10). Se consideraron como sueros positivos aquellos que mostraron valores de absorbancias iguales o mayores de 0.421 .

\section{TESA-BLOT}

Con la finalidad de establecer reacciones cruzadas o coinfecciones entre $L$. infantum y T. cruzi, se llevó a cabo la prueba WB usando los antígenos recombinantes TESA de $T$. cruzi, los cuales son reconocidos exclusivamente por sueros que han estado en contacto con este parásito (14). Esta prueba se desarrolló por medio de un trabajo colaborativo con la Dra. Eufrosina S. Umezawa, investigadora del Instituto de Medicina Tropical de Sao Paulo, Universidad de Sao Paulo, Brasil.

\section{Análisis Estadístico}

Se construyó una base de datos en EXCEL 6.0. Para la validación estadística de los resultados se utilizó la estadística descriptiva; y la concordancia de las pruebas se efectuó con el test de Kappa usando el programa WIN EPISCOPE 2.0 (Borland ${ }^{\circ}$ \& Delphi TM, 1998).

\section{RESULTADOS}

La población estudiada estuvo conformada por perros mestizos ( $\mathrm{n}=211)$, con una edad promedio entre 1 y 2 años. El 55,5 \% machos y el 44,5 \% hembras.

La seroreactividad ante antígenos totales de L. infantum en el grupo fue del 44,1 \% (93/211) y 50,2 \% (103/211) mediante IFAT y ELISA, respectivamente. La concordancia entre las dos técnicas diagnósticas fue baja (Índice de Kappa del 12,1\%). Los resultados detallados por municipio se presentan en la Tabla 1.

La reactividad de los sueros evaluados ante antígenos recombinantes específicos de T. cruzi usando WB, fue del 1,42 \% (3/211). Sólo dos de los tres 
sueros positivos, presentaron reactividad ante antígenos totales de L. Infantum, por IFAT y ELISA.

Los signos clínicos que se presentaron con mayor frecuencia fueron: onicogrifosis (36,4\%), caquexia (26,9\%) y alopecia (23,1\%). La linfadenitis, las úlceras cutáneas y los trastornos digestivos, se presentaron en orden decreciente (7,7 \%, 3,8 \% y 3,8 \% respectivamente). En la Tabla 2 se presenta la relación entre los signos clínicos y la seropositividad a IFAT y ELISA. Las razones de prevalencia entre los resultados de la evaluación clínica de los animales y la seropositividad a las dos pruebas, fue baja $(0,89$ y 0,85 respectivamente).

Tabla 1. Seroprevalencia de leishmaniasis canina en el grupo evaluado por las técnicas IFAT y ELISA. Tolima, 2006

\begin{tabular}{lccccc}
\hline Municipio & \multicolumn{2}{c}{ IFI } & \multicolumn{4}{c}{ ELISA } \\
& Positivos & $\%$ & Positivos & $\%$ & Total \\
\hline Ataco & 9 & 36,0 & 10 & 40,0 & 25 \\
Coello & 8 & 30,8 & 16 & 61,5 & 26 \\
Coyaima & 10 & 37,0 & 17 & 63,0 & 27 \\
Natagaima & 7 & 35,0 & 17 & 85,0 & 20 \\
Ortega & 41 & 64,1 & 26 & 40,6 & 64 \\
Purificación & 18 & 36,7 & 20 & 40,8 & 49 \\
\hline Positiva: Títulos superiores a 1:32 IFI & Absorbancias: $>0,421$ ELISA
\end{tabular}

Tabla 2. Estado clínico de los animales seropositivos evaluados por las diferentes pruebas diagnósticas

\begin{tabular}{lcccc}
\hline \multirow{2}{*}{ Estado clínico } & \multicolumn{2}{c}{ IFI } & \multicolumn{2}{c}{ ELISA } \\
& Positivos & $\%$ & Positivos & $\%$ \\
\hline Asintomático & 40 & 43,0 & 43 & 41,7 \\
Oligosintomático & 50 & 53,7 & 57 & 55,4 \\
Polisintomático & 3 & 3,3 & 3 & 2,9 \\
Total & 93 & & 103 & \\
\hline
\end{tabular}

\section{DISCUSIÓN}

Los caninos han sido descritos como importantes reservorios de L infantum en América Latina y probablemente juegan un papel esencial en la transmisión de LV, teniendo en cuenta que la presencia de perros seropositivos es considerado como un posible factor de riesgo para la infección por L. infantum en el hombre $(1,5,6,15)$. Consecuentemente, el diagnóstico precoz de la LVC, es utilizado como medida de control para reducir la transmisión de la infección de los reservorios al hombre (15). En Colombia, el diagnóstico de L. infantum en la población canina ubicada en las zonas endémicas, se viene realizando tradicionalmente por IFAT y recientemente por ELISA. 
La seroprevalencia de la infección por Leishmania infantum en el grupo de caninos evaluados por medio de las pruebas diagnósticas IFAT y ELISA (44,1 \% y 50,2 \% respectivamente) fueron más altas que las encontradas en estudios similares realizadas en Colombia por IFAT: Neiva (Huila), 17,2 \%; Córdoba, 16 \%; Cerro Vidales y Ovejas (Sucre), 3,8 \%; Rovira (Tolima), 12,5 \%; San Andrés de Sotavento (Córdoba) y Ovejas (Sucre), 9,6 \% (11). Se observó además una alta reactividad de los sueros caninos procedentes de diferentes municipios del Tolima (Tabla 1), en donde se reportan anualmente casos de LV en niños menores de dos años principalmente.

La prueba TESA blot usada para discriminar reacciones cruzadas con $T$. cruzi, contiene antígenos específicos de trypomastigotes que muestran una alta sensibilidad no sólo en los casos de enfermedad de Chagas crónica, sino también en la enfermedad aguda y congénita $(11,16,17)$. Esta detectó sólo 3 sueros positivos, que corresponden a una seropositividad del 1,42 \% (3/211), mas baja que la reportada por Montenegro (17) en Costa Rica (27,7 \% mediante ELISA); Gurtler (19) y Diosque (20) en Argentina (41,2 \% por xenodiagnóstico y 15,1\% por serología y examen parasitológico, respectivamente) y Estrada-Franco (21) en Mexico (17,5 \% usando cinco pruebas serológicas). Sin embargo, fue similar a la establecida por Shadomy (22) en Texas (2,6 \% usando diferentes técnicas diagnósticas) y por Barr (23) en Louisiana (4,7\% por ELISA).

Es importante destacar la débil correlación entre los resultados obtenidos con las dos técnicas usadas que emplearon antígeno colombiano (Índice de Kappa=12,1 \%), las cuales identificaron como positivos diferentes grupos de animales, aspecto que se podría explicar porque el tratamiento para obtener los antígenos fue diferente en las dos técnicas, siendo posible identificar un grupo distinto de epitopes (24); o se puede deber a sus diferentes valores de sensibilidad y especificidad, aspectos que favorecen la sobreestimación de la infección y la presencia de resultados falsos positivos, por la reacción cruzada con otros patógenos $(4,5,24,25)$.

Los hallazgos del presente estudio ponen de manifiesto que se estaría llevando al sacrificio a caninos no infectados, pero también que no se estaría detectando una proporción de animales infectados, que se convierten en fuentes de infección para la población canina y humana (26). Todo esto, desde luego, interfiere en la evaluación del impacto de las medidas de control en salud pública que deberían mostrar una disminución en cifras ya sea de la incidencia o de la prevalencia de LVZ $(1,27,28)$. 
Las pruebas IFAT y ELISA usando antígeno colombiano presentaron mayor reactividad y capacidad para identificar animales oligosintomáticos y asintomáticos (Tabla 2), observándose que los animales polisintomáticos tuvieron menor reactividad, resultados que concuerdan con los descritos por Pinelli (29) y Moreira (30), quienes detectaron inmunosupresión en la producción de anticuerpos contra Leishmania en perros sintomáticos. Así mismo, las razones de prevalencia $(\mathrm{RP}<1)$, demostraron que no hay ninguna asociación entre la ocurrencia de manifestaciones clínicas y la seropositividad a las diferentes pruebas, sugiriéndose que la información clínica no ofrece ninguna ayuda para discriminar infecciones caninas por L. infantum, por lo tanto este criterio no se debe tener en cuenta para la identificación de animales susceptibles a sacrificar en los programas de control en las zonas endémicas.

Para el diagnóstico de la LVC se requiere de pruebas eficientes, que no subestimen la prevalencia de la enfermedad, y que permitan obtener resultados confiables, que minimicen las reacciones falsas positivas y la reacción cruzada con otros agentes patógenos $(1,5,15)$. La baja asociación entre los resultados obtenidos con las dos técnicas usadas (IFAT y ELISA con antígeno colombiano), permiten sugerir que desde la óptica de la salud pública, la carencia de un método confiable para el diagnóstico adecuado de la LVC, puede inducir a que la eliminación de caninos seropositivos no contribuya para interrumpir la transmisión de LV en los focos ecoepidemiológicos en Colombia. Haciendo necesario además, el desarrollo de estudios posteriores, que evalúen diferentes epitopes y establezcan un algoritmo de pruebas diagnósticas para confirmar el estado real de la infección en animales natural y experimentalmente infectados, como lo han reportado otros investigadores en Colombia (10); aspecto fundamental para orientar eficientemente las políticas y recursos de salud pública tendientes al control de la enfermedad •

Agradecimientos. Los autores quieren expresar su agradecimiento a la Secretaría Departamental de Salud del Tolima, Colombia, por el financiamiento de esta investigación y a la Maestría en Ciencias Biológicas de la Universidad del Tolima.

\section{REFERENCIAS}

1. Alves WA, Bevilacqua PD. Quality of diagnosis of canine visceral Leishmaniasis in epidemiological surveys: an epidemic in Belo Horizonte, Minas Gerais, Brazil, 1993 - 1997. Cad. Saude Pública 2004; 20 (1):259 - 265.

2. Ferroglio, F, Centaro E, Mignone W, Trisciuoglio A. Evaluation o fan ELISA rapid device for the serological diagnosis of Leishmania infantum infection in dog as compared with immunofluorescence assay and Western blot. Veterinary Parasitology 2007; 144:162166. 
3. Savani E. Schimonsky BV; Camargo OMC, D'auria SR. Surveillance of American visceral Leishmaniasis in dogs from a non-endemic area, Brazil. Revista Saude Pública 2003; 37(2):260-262.

4. Cabrera MA, Paula A, Camacho LA, Marzochi, MC, Xavier SC, Da Silva AV, CansenAM. Canine Visceral Leishmaniasis in Barra de Guaratiba, Rio de Janeiro, Brazil: Assesment of risk factors. Rev. Institute Medicine Tropical Sao Paulo 2003; 45(2):79-83.

5. Silva ES, Gontijo MF, Pacheco RS, Fiuza VOP, Brazil RP. Visceral Leishmaniasis in the Metropolitan Region of Belo Horizonte, State of Minas Gerais, Brazil, Mem Inst Oswaldo Cruz 2001, 96(3):285-291.

6. Travi, B. Un enfoque integral al estudio de animales reservorios, con énfasis en las Leishmaniosis del Nuevo mundo. Médicas UIS 1998, 12:311-315.

7. Marzochi MC, Marzochi KBF. Tegumentary and Visceral Leishmaniasis in Brazil-Emerging Antropozoonosis and Possibilities for their control. Cad. Saude Pública. 10(supplement 2):359-375.

8. Mettler M, Grimm F, Capelli G, Camp H, Deplazes P. Evaluation of Enxyme-Linked Immunosorbent assays, an Immunofluorescent-antibody test, and two rapid tests (Immunochromatographic-Dipstick and gel tests) for serological diagnosis of symptomatic and asymptomatic Leishmania infections in dogs. Journal of Clinical Microbiology 2005; 43(11):5515-157.

9. Travi B, Tabares C, Cadena H, Ferro C, Osorio Y. Canine Visceral Leishmaniasis in Colombia: relationship between clinical and parasitologic status and infectivity for sand flies. The Amercian Society of Tropical Medicine and Higiene 2001; 64(3,4):119-124.

10. Vega JC, López MC, Vargas J, Ayala M, Nicholls S, Bello F, Fernandez J, Escobar J. Estandarización de la prueba de ELISA para el inmunodiagnóstico de la Leishmaiosis visceral canina. En: Agudelo C.( ed.) I Encuentro de Investigadores en Salud Pública de la Universidad Nacional de Colombia. Bogotá: Instituto de Salud Pública; 2003.

11. Fernández J, Charry T, Bello F, Escobar J, Lozano C, Ayala M, Nicholls R, Vargas J, Moncada L, Corredor A, López MC. Prevalencia de Leishmaniosis Visceral canina en municipios de Huila Colombia. Rev. Salud pública 2002; 4(3):278-285.

12. Instituto Colombiano Agustín Codazzi, Diccionario geográfico de Colombia. Bogotá D.C.; 1996.

13. Corredor A, Alvarez CA, Agudelo CA, Bueno M, López MC, Cáceres E, Reyes P, Duque S, Gualdrón LE, Santacruz M. Prevalence of Trypanosoma cruzi and Leishmania chagasi infection and risk factors in a Colombian indigenous population. Rev. Inst.Med. trop. Sao Paulo 1999; 41(4):229-234.

14. Umezawa E, Bastos S, Camargo M, Yamauchi L, Santos M, Gonzalez A, Zingales B, Levin M, Sousa O, Rancel-Aldao R, Silveira J. Evaluation of recombinant antigens for serodiagnosis of Chagas' disease in South and Central America. Journal of Clinical Microbiology 1996; 37:1554-1560.

15. Téran-Angel G, Schallig H, Zerpa O, Rodríguez V, Ulrich M, Cabrera M. The direct agglutination test as an alternative method for the diagnosis of canine and human visceral leishmaniasis. Biomédica 2007; 27:447-53.

16. Nakazawa M, Rosa D, Pereira V, Moura M, Furtado V, Souza W, Das Neves M, Barros S, Abath F, Gomes Y. Excretory-secretory antigens of Trypanosoma cruzi are potentially useful for serodiagnosis of Chronic Chagas' disease. Clinical and Diagnostic Laboratory Immunology 2001; 8(5):1024-1027.

17. Umezawa E, Luquetti A, Levitus G, Ponce C, Ponce E, Henríquez D, Revollo S, Espinoza B, Sousa O, Khan B, Da Silviera JF. Serodiagnosis of chronic and acute Chagas' disease with Trypanosoma cruzi recombinant proteins: results of a collaborative study in six Latin American countries. Journal of clinical Microbiology 2004; (42):449-452.

18. Montenegro V, Jiménez M, Pinto Dias Jc, Zeledón R. Chagas disease in dogs from endemic areas of Costa Rica. Mem Inst Oswaldo Cruz 2002; 97(4):491-494. 
19. Gurtler R, Cohen J, Cecere M, Lauricella M, Chuit R, Segura E. Influence of humans and domestic animals on the household prevalence of Trypanosoma cruzi in Triatoma infestans populations in northwest Argentina. Am.J. Trop.Med. Hyg. 1998; 58(6):748758.

20. Diosque P, Padilla A, Cimino R, Cardozo R, Sanchez O, Marco J, Zacca R, Meza C, Juarez A, Rojo H, Rey R, Corrales R, Nasser J, Basombrío M. Chagas disease in rural areas of Chaco province Argentina: epidemiologic survey in humans, reservoirs, and vectors. Am. J. Trop. Med. Hyg 2004; 71(5):590-593.

21. Estrada-Franco JG, Bhatia V, Diaz-Albiter H, Ochoa-Gracía L, Barbosa A, Vazquez-Chagoyan Jc, Martines-Péres Ma, Guzman-Bracho C, Garg N. Human Trypanosoma cruzi infection and seropositivity in dogs, Mexico. Emergeting Infection Disease 2006; 12(4):624-630.

22. Shadomy S, Waring SC, Chappell C. Combined use of Enzime-linked Immunoabsorbent assay and flow cytometry to detect antibodies to Trypanosoma cruzi in domestic canines in Texas. Clinical and Diagnostic Laboratory Immunology 2004; 3:313-319.

23. Barr SC, Dennis VA, Klei Tr. Serologic and blood culture survey of Trypanosoma cruzi infection in four canine population of southern Lousiana. Am J Vet Res 1991; 52(4):570573.

24. Iniesta L, Fernández-Barredo S, Bulle B, Gómez T, Piarroux R, Gállego M, Alunda J, Portús M. Diagnostic techniques to detect Cryptic Leishmaniasis in dogs. Clinical and Diagnostic Laboratory Immunology 2002; 9( 5):1137-1141.

25. Barrouin-Melo S, Larangeira D, Filho F, Trigo J, Silva JF, Franke C, Aguiar P, Dos Santos W, Carvalho L. Can splen aspirations be safely used for the parasitological diagnosis of canine visceral leishmaniosis? A study on asymptomatic and polysimptomatic animals. The Veterinary Journal 2004; 171, $331-339$.

26. Solano-Gallego L; Morell P, Arboix M, Alberola J, Ferrer LL. Prevalence of Leishmania infantum infection in dogs living in an area of canine Leishmaniasis endemicity using PCR on several tissues and serology. Journal of Clinical Microbiology 2001; (2):560-563.

27. Palatnik-De-Sousa CB, Wania R, Franca-Silva, Da Costa RT, Reis BA, Palatnik M, Mayrink W, Genaro O. Impact of canine control on the epidemiology of canine and human visceral Leishmaniasis in Brazil. The American Society of Tropcial Medicine and Hygiene 2001; 65(5):510-517.

28. Ferreira EDC, de Lana M, Carneiro M, Reis AB, Paes DV, da Silva ES, Schallig H, Gontijo CMF. Comparison of serological assays for the diagnosis of canine visceral leishmaniasis in animals presenting different clinical manifestations. Veterinary Parasitology 2007; (146):235-241.

29. Pinelli E, Killick-Kendrick R, Wagenaar J, Bernadina W, Del Real G, Ruitenberg J. Cellular and Humoral immune responses in dogs experimentally and naturally infected with Leishmania infantum. Infection and immunity 1994; 62: 229-235.

30. Moreira MAB, Luvizotto MCR, Garcia JF, Corbett CEP, Laurenti MD. Comparison of parasitological, immunological and molecular methods for the diagnosis of Leishmaniasis in dogs with different clinical signs. Veterinary parasitology 2007; 145:245-252. 